\title{
Desiderata: Soziale Netzwerk und gesundheitliche Ungleichheiten - welche Fragen bleiben offen?
}

\author{
Olaf Reis, Philip Adebahr, Stefan Brandt, Lea Ellwardt, \\ Markus Gamper, Laura Hoffmann, Sylvia Keim-Klärner, \\ Andreas Klärner, André Knabe, Gerhard Krug, \\ Annett Kupfer, Daniel Lois, Martin Mlinarić, \\ Irene Moor, Britta Müller, Mathilde Niehaus, \\ Nancy Reims, Matthias Richter, Julia Seidel, \\ Holger von der Lippe, Nico Vonneilich \\ und Stefan Zapfel
}

O. Reis $(\bowtie) \cdot$ A. Knabe $\cdot$ B. Müller

Rostock, Deutschland

E-Mail: olaf.reis@med.uni-rostock.de

A. Knabe

E-Mail: andre.knabe@uni-rostock.de

B. Müller

E-Mail: britta.mueller@med.uni-rostock.de

P. Adebahr

Chemnitz, Deutschland

E-Mail: philip.adebahr@soziologie.tu-chemnitz.de

S. Brandt $\cdot$ H. von der Lippe

Berlin, Deutschland

E-Mail: brandt@tu-berlin.de

H. von der Lippe

E-Mail: holger.vonderlippe@ medicalschool-berlin.de

L. Ellwardt · M. Gamper · M. Niehaus

Köln, Deutschland

E-Mail: ellwardt@wiso.uni-koeln.de

(C) Der/Die Herausgeber und der/die Autor(en) 2020

A. Klärner et al. (Hrsg.), Soziale Netzwerke und gesundheitliche Ungleichheiten, https://doi.org/10.1007/978-3-658-21659-7_17 
M. Gamper

E-Mail: m.gamper@uni-koeln.de

M. Niehaus

E-Mail: mathilde.niehaus@uni-koeln.de

L. Hoffmann · M. Mlinarić · I. Moor

Halle (Saale), Deutschland

E-Mail: laura.hoffmann@medizin.uni-halle.de

M. Mlinarić

E-Mail: martin.mlinaric@medizin.uni-halle.de

I. Moor

E-Mail: irene.moor@medizin.uni-halle.de

S. Keim-Klärner · A. Klärner

Braunschweig, Deutschland

E-Mail: sylvia.keim-klaerner@thuenen.de

A. Klärner

E-Mail: andreas.klaerner@thuenen.de

G. Krug $\cdot$ N. Reims $\cdot$ S. Zapfel

Nürnberg, Deutschland

E-Mail: gerhard.krug@iab.de

N. Reims

E-Mail: nancy.reims@iab.de

S. Zapfel

E-Mail: stefan.zapfel@ifes.uni-erlangen.de

A. Kupfer · J. Seidel

Dresden, Deutschland

E-Mail: annett.kupfer@tu-dresden.de

J. Seidel

E-Mail: julia.seide14@tu-dresden.de

D. Lois

Neubiberg, Deutschland

E-Mail: daniel.lois@unibw.de

M. Richter

Halle, Deutschland

E-Mail:m.richter@medizin.uni-halle.de

N. Vonneilich

Hamburg, Deutschland

E-Mail: n.vonneilich@uke.de 


\section{$1 \quad$ Einleitung}

„Sag mir, wie viel Deine Freunde verdienen, und ich sage Dir, ob Du rauchst, welche Krankheiten Du hast und wie alt Du werden wirst!" Mit dieser etwas zugespitzten Aussage wurde am Anfang dieses Bandes vorgeschlagen, den empirisch gut bestätigten Zusammenhang von sozialen und gesundheitlichen Ungleichheiten aus der Perspektive der Netzwerkforschung zu beleuchten (siehe Kap. „Soziale Netzwerke und gesundheitliche Ungleichheiten - eine neue Forschungsperspektive"). Soziale Netzwerke werden dabei als vermittelnde Instanz auf einer intermediären oder Meso-Ebene verstanden, deren Struktur und Funktion zwischen vertikalen (Einkommen, Bildung, berufliche Stellung etc.) sowie horizontalen (z. B. Alter, Geschlecht, ethnische Herkunft) und gesundheitlichen Ungleichheiten (z. B. Lebenserwartung, Erkrankungsraten) vermittelt (Mediatorfunktion) oder deren Beziehung beeinflusst (Moderatorfunktion).

Damit wird der Versuch unternommen, zwischen die makro- und die mikrofundierte Gesundheitswissenschaft gewissermaßen eine „mesofundierte“ Forschung zu stellen, die notwendigerweise die Makro- und die Mikro-Perspektiven vereint. Mit diesem Anspruch verortet sich die Netzwerkforschung zwischen klassischen makrosoziologischen Ansätzen, die sich auf große Gruppen (z. B. soziale Klassen, Geschlechter) beziehen, und mikrofundierten Ansätzen, die individuelle gesundheitliche Voraussetzungen, Präferenzen und Verhaltensweisen betonen. Sie greift damit auch die Kritik von Emirbayer (1997) auf, der auf der einen Seite allzu einfache Modelle rationaler und am Eigeninteresse orientierter Akteure kritisiert; auf der anderen Seite aber auch Ansätze bemängelt, die den strikten Vorrang von Normen oder gesellschaftlichen Strukturen behaupten, welche das Handeln von Subjekten „leiten“ (vgl. Klärner und Keim 2019). Im sozialen Netzwerk treffen individuelle Voraussetzungen, wie die genetische Ausstattung, die Persönlichkeit, Vorlieben etc., auf die soziale „Strukturation“ (Giddens 1984) durch vertikale und horizontale Ungleichheiten. Soziale Netzwerke werden einerseits vom Individuum beeinflusst, andererseits wirken sich Netzwerkveränderungen auf das Individuum aus.

Für die Klärung der Zusammenhänge zwischen individueller Gesundheit, Netzwerk und sozialer Ungleichheit bedarf es einiger Voraussetzungen, denen sich der erste Teil dieses Bandes widmet. In der wissenschaftlichen Auseinandersetzung mit den Themen rund um soziale Beziehungen, soziales Kapital und soziale Netzwerke kann festgestellt werden, dass die unterschiedlichen Begriffe nicht klar voneinander abgegrenzt und teilweise synonym verwendet werden. Damit bleiben die Begriffe oft unklar, was eine einheitliche Definition und ein 
einheitliches Verständnis sozialer Beziehungen und sozialer Netzwerke erschwert. Um zu einem klareren Verständnis beizutragen, unternimmt Nico Vonneilich eine Einordnung der Begriffe und Konzepte (siehe Kap. „Soziale Beziehungen, soziales Kapital und soziale Netzwerke“). Markus Gamper (siehe Kap. „Netzwerktheorie(n)") schlägt in seinem Beitrag vor, Netzwerke primär strukturell zu verstehen, als Menge von Knoten (Akteuren auf unterschiedlicher Aggregatsebene), die durch Kanten (Beziehungen verschiedener Art) verbunden sind, über die ein Austausch (von Informationen, Emotionen, Gütern etc.) stattfindet. Soziales Kapital kann damit als Vorhandensein gesundheitlich ertragreicher Knoten und Kanten, soziale Unterstützung als Effekt dieser verstanden werden. Die Auftrittswahrscheinlichkeit ertragreicher Knoten und Kanten würde durch vertikale und horizontale Ungleichheiten moderiert, was sich auf der Mikroebene der Individuen in vor- oder nachteiligen Gesundheitseffekten (bezüglich Morbidität und Mortalität sowie dem subjektiven Gesundheitsempfinden), und auf der Makroebene in sozial ungleich verteilten psychischen und physischen Gesundheitszuständen, d. h. gesundheitlichen Ungleichheiten, niederschlagen sollte.

Neben sozialem Kapital bzw. sozialer Unterstützung werden in dem Beitrag von Andreas Klärner und Holger von der Lippe weitere mögliche Wirkmechanismen in sozialen Netzwerken diskutiert: soziale Integration, sozialer Einfluss und (soziale) Ansteckung (siehe Kap. „Wirkmechanismen“). Diese Konzepte sind, wie der Beitrag zeigt, als Sammelbegriffe heuristisch durchaus nützlich, während ein allgemeines, sparsames und trennscharfes theoretisches Modell, in dem die möglichen Zusammenhänge präzise definiert und differenziert werden können, weiterhin aussteht. Zukünftige Forschung steht den Autoren zufolge vor der Aufgabe, die Unterscheidungen von 1) direkten und indirekten sowie 2) positiven und negativen Gesundheitseffekten durch 3) verschiedene Akteure oder Sektoren von Netzwerken zu berücksichtigen und diese Aspekte für unterschiedliche Krankheitsrisiken differenziert zu analysieren.

In der Literatur, das machen die Autor*innen der einzelnen Beiträge deutlich, wird der Netzwerk-Begriff noch zu häufig lediglich als Metapher gebraucht, steht z. B. für die Kontakthäufigkeit zu Eltern und Freunden oder für soziale Unterstützung, wird dabei aber nicht strukturell operationalisiert. Netzwerkstudien im oben ausgeführten strukturellen Verständnis sind im Bereich der Erforschung gesundheitlicher Ungleichheiten noch relativ selten anzutreffen. Auch weisen die Autor*innen der Beiträge dieses Bandes darauf hin, dass hier noch ein erheblicher theoretischer wie auch methodologischer Entwicklungsbedarf besteht. Damit das Konzept der sozialen Netzwerke die geforderte „Mesofundierung“ der Gesundheitswissenschaften zwischen Makro- und Mikroprozessen leisten kann, 
bedarf es einer Weiterentwicklung der vorliegenden theoretischen Modelle (siehe Kap. „Soziale Netzwerke und gesundheitliche Ungleichheiten“) und einer konsequenten Anwendung der Methoden der Netzwerkanalyse (siehe Kap. „Netzwerkanalyse"). So gilt es zum Beispiel, gesundheitliche Risiken und Ressourcen im Netzwerk zwar als Bestandteil der Lebenswelt, aber auch als relativ unabhängig vom Einzelnen zu sehen. Beispielsweise ist die Exposition gegenüber rauchenden Mitschülern für Jugendliche aus niedrigeren Einkommensschichten größer, unabhängig davon, ob sie selbst rauchen oder nicht. Diesen Übergang leistet die Gesamtnetzwerkanalyse, in die innerhalb eines definierten Raumes alle Knoten und Kanten eingehen. Die Akteure (als Knoten) gehen Beziehungen auch ohne Zutun oder Wissen des Einzelnen ein (Alteri-Kanten). Dabei haben diese (teils vielleicht unbewussten) Beziehungen ihrerseits wieder Auswirkungen auf Knoten und Kanten im egozentrierten Netzwerk, die sich dann auf die individuelle Gesundheit auswirken können. Weiter ermöglichen es qualitative und egozentrierte Netzwerkverfahren aber auch, den Raum möglicher Einflüsse des sozialen Netzwerkes auf Gesundheit durch Hinzunahme bisher unbekannter Akteure (Knoten) durch sogenannte Akteursgeneratoren zu erweitern. Dabei stellen alle Autor*innen dieses Bandes jedoch übereinstimmend fest, dass das Feld der Netzwerkforschung in der Medizin- und Gesundheitssoziologie sowie in der Sozialepidemiologie große Lücken aufweist, sowohl was die Berücksichtigung von Alteri-Beziehungen als auch was die die Hinzunahme möglicher Akteure betrifft.

Eine erhebliche strukturelle Erweiterung der Netzwerkforschung wird von Philip Adebahr (siehe Kap. „Negative Beziehungsaspekte und gesundheitliche Ungleichheiten“) angemahnt - der Einbezug sogenannter negativer Beziehungen (negative relationships) oder präziser ausgedrückt: negativer Beziehungsaspekte (negative ties). Dabei sollten Beziehungsaspekte, die mit Streit, Konflikt, psychischen Belastungen oder physischen Verletzungen verbunden sind, nicht nur als Risiken für Gesundheit verstanden werden; vielmehr ist ihre Wirkung vom Kontext anderer Beziehungen - des gesamten Netzwerkes - abhängig. Ebenso wenig sollte das Konzept der negativen Beziehungen auf Ego-Netzwerke beschränkt werden. Möglicherweise werden moderierende oder mediierende Funktionen des Netzwerkes, wie sie oben formuliert wurden, nur unter Einbezug negativer Beziehungen in Alteri-Beziehungen besser verstehbar und erklärbar. Beispielsweise könnten Limitierungen des Netzwerkes aufgrund eines geringeren Einkommens möglicherweise durch das Vorhandensein von negativen Beziehungen besser beschrieben werden als durch das Nicht-Vorhandensein positiver Beziehungen. Hier klafft eine Forschungslücke, denn bisher ist zu negativen Beziehungsaspekten nicht ausreichend bekannt, inwiefern sie beispielsweise Netzwerke organisieren, inwiefern sie sozial ungleich verteilt sind oder inwiefern 
es Ansteckungsmechanismen gibt, die von vertikalen Ungleichheitsdimensionen abhängen. Sozialer Aufstieg beispielsweise ließe sich nicht nur als Zugewinn positiver Beziehungsaspekte darstellen, sondern auch als die Loslösung von negativen Beziehungsaspekten. Der Einbezug negativer Aspekte sozialer Beziehungen ähnelt in einigen Aspekten anderen Konzepten aus der Beziehungsforschung, beispielsweise dem Konzept der ,intergenerationalen Ambivalenz“ (Lüscher und Pillemer 1998).

Unabhängig von den eben beschriebenen theoretischen und methodischen Herausforderungen an die Netzwerkforschung sollen im Folgenden inhaltliche Lücken aufgelistet werden, wie sie in den Kapiteln dieses Bandes beschrieben werden. Die Beiträge des Bandes stellen den Forschungsstand auf verschiedenen Feldern dar. Diese Forschungsfelder werden einmal entlang der Phasen des Lebenslaufs strukturiert, d. h. von der Kindheit bis ins hohe Alter. Eine zweite Struktur folgt ausgewählten Dimensionen vertikaler und horizontaler Ungleichheiten - vom sozioökonomischen Status bis hin zu natio-ethno-kultureller Zugehörigkeit. Im Folgenden werden wir nun offen gebliebene Fragen aus Sicht der Lebenslaufforschung und aus Sicht der Ungleichheitsforschung benennen und hoffen, damit eine Orientierung für zukünftige Forschungsanstrengungen geben zu können. Um es vorwegzunehmen: Studien, die Gesundheit, Netzwerk und soziale Ungleichheit im Sinne eines einheitlichen Modells zusammenführen, sind nicht vorhanden. Damit wird eine Erwartung an den vorliegenden Band, umfassende Ergebnisse zu ebendiesen Wechselwirkungen zu präsentieren, notwendigerweise enttäuscht.

\section{Offene Fragen aus Sicht der Lebenslaufforschung}

Zur Lebenslaufperspektive verweisen unterschiedliche Autor*innen im zweiten Teil dieses Bandes darauf, dass fast alle bekannten Studien biologische Faktoren aus den Modellen ausklammern (siehe Kap. „Soziale Netzwerke, familiales Sozialkapital und kindliche Gesundheit" und "Soziale Netzwerke und gesundheitliche Ungleichheiten im jungen und mittleren Erwachsenenalter"). Das betrifft insbesondere genetische Faktoren, die nicht nur in der Kindheit, sondern lebenslang wirken und oft in Interaktion mit umweltlichen Ereignissen stehen. Derartige Ereignisse können auch direkt netzwerkbezogen sein, wie etwa Verluste zentraler Beziehungen oder Veränderungen der Positionen im Netzwerk. Dieses Thema ist weitgehend wissenschaftliches Neuland und bedarf großer Studien mit mehrdimensionaler Datenlage. In anderen Worten: Es bedarf vollständiger biopsycho-sozialer Erhebungen (siehe Abschn. 5). 
Weiterhin scheinen unterschiedliche Mechanismen sozialer Netzwerke (siehe Kap. „Wirkmechanismen in sozialen Netzwerken“) in verschiedenen Lebensphasen zu wirken. Beispielsweise dürfte sozialer Einfluss in Form direkter sozialer Kontrolle, zu Beginn des Lebens für die Individualentwicklung (ausgeübt von den Eltern) erheblicher sein als soziale Ansteckung. Soziale Ansteckung dürfte mit zunehmender Autonomie des Individuums im Lebensverlauf eher zunehmen, um möglicherweise zum Lebensende hin gegenüber sozialer Kontrolle (z. B. ausgeübt von Helfern) wieder an Bedeutung zu verlieren. Um diese zeitliche Perspektive der Dynamiken und Veränderungen sozialer Netzwerke zu erforschen, bedarf es weiterer konzeptueller Zusammenschlüsse von soziologischer Netzwerk- und psychologischer Entwicklungsforschung, in denen verschiedene phasenspezifische Regulationsmechanismen angenommen werden.

Für die Netzwerke von Kindern stellt Daniel Lois (siehe Kap. „Soziale Netzwerke, familiales Sozialkapital und kindliche Gesundheit") fest, dass in der empirischen Forschung Familiennetzwerke häufig zu eng gefasst werden und Geschwister, Großeltern, entferntere Verwandte oder optierte Familienmitglieder zu selten eingeschlossen werden. Die sich im Zuge der Modernisierung diversifizierenden Familiennetzwerke würden darüber hinaus vom Einbezug der Alteri-Kanten und deren Formalisierung auch als negative Beziehungen profitieren. So sind Netzwerke von Kindern aus Patchwork-Familien denkbar, die vier oder mehr Paare von Großeltern enthalten, deren Alteri-Beziehungen möglicherweise von den nicht immer konfliktfreien Beziehungen der zugehörigen Eltern, etwa nach einer Scheidung (siehe Kap. „Soziale Netzwerke und gesundheitliche Ungleichheiten im jungen und mittleren Erwachsenenalter"), beeinflusst werden. Die Muster derartiger positiver und negativer Beziehungen von entfernteren Verwandten werden in vielerlei Gestalt relevant für die Gesundheit des Kindes, etwa, wenn bestimmte Großeltern (beispielsweise Eltern des geschiedenen Partners) aufgrund ihres riskanten Gesundheitsverhaltens aus dem Netzwerk eigentlich ausgeschlossen werden (von der Mutter), aufgrund negativer Beziehungen zum nachfolgenden Partner jedoch als Ressource finanzieller oder praktischer Unterstützung benötigt werden.

Das Jugendalter ist unter der Netzwerkperspektive das bisher am besten untersuchte (siehe Kap., ,Soziale Netzwerke, Gesundheit und gesundheitliche Ungleichheiten im Jugendalter"). Hier werden von Irene Moor und Ko-Autor*innen die meisten und größten, methodisch fortgeschrittenen Studien vorgestellt, die dennoch inhaltliche Lücken aufweisen. Ein Großteil der zitierten Studien im Jugendalter richtet sich überwiegend auf schulische Kontexte. Damit wird zu häufig auf wichtige Netzwerkanteile von Familien und außerschulischen Kontakten (z. B. in Vereinen) zugunsten von Erhebungen des Gesamtnetzwerkes (Schule hat 
klar definierte Grenzen) verzichtet. Da die meisten Studien ein querschnittliches Design haben, sind kausale Aussagen selten möglich. Dennoch kommen die Studien zum Jugendalter dem Ziel, Netzwerk und gesundheitliche Ungleichheiten zusammenzudenken, relativ zu Studien aus anderen Lebensabschnitten am nächsten. Die meisten Studien konzentrieren sich auf riskantes Gesundheitsverhalten, vor allem den Nikotingebrauch. Studien zur psychischen Gesundheit gibt es nur in Ansätzen, die so alarmierend wie vielversprechend sind. So fungieren soziale Netze, insbesondere Freundesgruppen, als Mediatoren zwischen dem sozioökonomischen Status von Jugendlichen und ihrem Risikoverhalten. Allerdings sind bislang nur wenige Forschungsarbeiten bekannt, die die Bedeutung der sozialen Netzwerke für gesundheitliche Ungleichheiten (fernab des Tabakkonsums) untersuchen.

Die Assoziation von Netzwerk und Gesundheit im Erwachsenenalter scheint von zwei Faktoren beeinflusst: von lebenslangen Konstanten (etwa verlängerten Freundesnetzwerken oder der Persönlichkeit des Erwachsenen) und biografischen Transitionen, die mit Veränderungen im Netzwerk einhergehen. Nur wenige der referierten Studien betrachten diese Zusammenhänge im Kontext vertikaler Ungleichheiten, obgleich etliche Ereignisse diesen Zusammenhang nahelegen, etwa bei Scheidungen. Holger von der Lippe und Olaf Reis (siehe Kap. „Soziale Netzwerke und gesundheitliche Ungleichheiten im jungen und mittleren Erwachsenenalter") stellen diesbezügliche Anforderungen an die Netzwerkforschung zusammen. Ereignisstrukturen (etwa ihre Reihenfolge) und -zeitpunkte im Lebensverlauf (timing) sollten in der Netzwerkforschung beachtet werden, da gleiche Ereignisse zu unterschiedlichen Zeitpunkten und in Verbindung mit anderen Ereignissen unterschiedliche Effekte auf Netzwerke haben und sich demnach unterschiedlich auf Gesundheit auswirken können. Darüber hinaus betonen die Autoren Effekte säkularen Wandels, der die Muster des Erwachsenseins nachhaltig beeinflussen kann, wobei vertikale Ungleichheiten eine nicht unwesentliche Rolle spielen.

Für das höhere Lebensalter zeigen Britta Müller und Lea Ellwardt (siehe Kap. ,Soziale Netzwerke und gesundheitliche Ungleichheiten im Alter") sowohl inhaltliche als methodische Lücken auf. Vorliegende Studien konzentrieren sich v. a. auf in eigener Häuslichkeit lebende Personen. Bislang ist unklar, inwiefern deren Befunde auch auf Bewohner von Pflegeeinrichtungen übertragbar sind. Auch der Effekt von individuellen Transitionen auf Netzwerke in den späten Lebensphasen ist eine noch offene Frage. $\mathrm{Zu}$ erwarten sind hierbei v. a. Einflüsse von gesundheitlichen Verschlechterungen und Funktionseinbußen. Bisherige Studien analysierten den Zusammenhang von SES, Gesundheit und sozialem Netzwerk vorrangig bezüglich des Krankheitsbildes Depression oder funktionaler 
und subjektiver Gesundheit. Die Frage, ob auch bei Demenzerkrankungen und schmerzassoziierten Erkrankungen Wechselwirkungen mit dem SES und sozialem Netzwerk auftreten, ist hingegen noch nicht geklärt. Die Autor*innen betonen, dass Forschungsdesigns, die komplexe Maße von sozialen Netzwerken im Alter erheben, für die Untersuchung der Beziehung von SES, Gesundheit und sozialem Netzwerk besser geeignet sind als einseitig quantitativ oder qualitativ ausgerichtete Instrumente. Bislang werden Netzwerkmerkmale in der gerontologischen Forschung meist nur indirekt, d. h. über sogenannte Proxy, gemessen. Das Potenzial etablierter Verfahren der Netzwerkanalyse sollte für die zukünftige Erforschung von Menschen im Alter wesentlich stärker genutzt werden.

\section{Offene Fragen aus Sicht der Ungleichheitsforschung}

Im dritten Teil des Bandes nähern sich die Autor*innen der Netzwerkforschung aus der Perspektive der Ungleichheitsforschung. Für Studien zum sozialen Status stellt Nico Vonneilich (siehe Kap., Sozialer Status, soziale Beziehungen und Gesundheit") fest, dass es kaum Untersuchungen gibt, die im Sinne von Mehr-Ebenen-Modellen makrosoziale, mikrosoziale und individuelle Prozesse verknüpfen. Nur selten werden diese Ebenen miteinander in Verbindung gebracht und die sozialstrukturellen Faktoren zur Entstehung oder der Verhinderung stabiler sozialer Netzwerke berücksichtigt und in Bezug zur Gesundheit gesetzt. Da es kaum einschlägige Studien mit umfangreichen Indikatorensätzen zu sozialen Netzwerken gibt, empfiehlt der Autor die Reanalyse vorhandener Datensätze, wie etwa der SHIP-Studie. Allerdings sind Sekundärdaten häufig mit Messproblemen behaftet - entweder auf der Ebene der Gesundheit oder des Netzwerkes. Auch für die relativ gut untersuchte Ungleichheit nach Erwerbslosigkeit (siehe Kap. „Arbeitslosigkeit, soziale Netzwerke und gesundheitliche Ungleichheit") beklagen Gerhard Krug und Ko-Autoren das Fehlen von Studien, die Netzwerke in ihrer Auswirkung auf gesundheitliche Ungleichheit zeigen. Dieses Fehlen ist umso bedauerlicher, da für dieses Ereignis gute Belege für netzwerkbezogene Mediator- und Moderatoreffekte erbracht wurden. Auch für dieses Thema lassen sich zeitliche Prozesse aufgrund des weitgehenden Fehlens von Längsschnittstudien kaum darstellen.

Insbesondere was psychische Gesundheit betrifft, kann bisher schlecht zwischen Ursache und Wirkung differenziert werden, zumal die meisten Studien auf ausgelesene Stichproben zurückgreifen. Derartige „Abwärtsspiralen“, in denen kleiner werdende Netzwerke und schlechtere Gesundheitslagen auftreten, sind 
beispielsweise für Alleinerziehende bisher nur im Ansatz gezeigt worden (siehe Kap. „Soziale Netzwerke und die Gesundheit von Alleinerziehenden“). Wie auch für die Erwerbslosigkeit gibt es für Alleinerziehende Belege, die auf Pufferwirkungen funktionaler Netzwerke hinweisen; differenziertere Analysen fehlen jedoch. Sylvia Keim-Klärner listet verschiedene Ansätze auf, die diesem Mangel abhelfen könnten, wobei sie zwischen Längsschnitt-, Querschnitt- und qualitativen Analysen unterscheidet. Insbesondere vom Einbezug negativer Beziehungen und Beziehungsinhalten verspricht sich die Autorin einen Wissenszuwachs, um der Komplexität und Ambivalenz von Beziehungskonfigurationen und Interaktionen gerecht zu werden.

Zum Thema Gender (siehe Kap. „Gender/Geschlecht und gesundheitliche Ungleichheiten"), einer zentralen Ungleichheitsdimension in der aktuellen sozialwissenschaftlichen Diskussion, stellen Markus Gamper und Ko-Autor*Innen fest, dass die Forschung fast ausschließlich mit den zwei Geschlechtsidentitäten „Mann“ und „Frau“ arbeitet. Im Zuge der gesellschaftlichen Debatte um Geschlechtsidentitäten jenseits der Zweigeschlechtlichkeit und der Entscheidung des Bundesverfassungsgerichtes aus dem Jahr 2017, welche den Gesetzgeber verpflichtet, eine dritte Option (,divers“) für intergeschlechtliche Personen in Geburten- und Einwohnermelderegistern vorzusehen, könnte der gesundheitlichen Lage auch von Personen mit anderen Geschlechtsidentitäten mehr Aufmerksamkeit gewidmet werden (vgl. Brand 2018). Über deren gesundheitliche Lage ist bislang, auch aufgrund der schwierigen Erfassung dieser Gruppe in repräsentativen Surveys, relativ wenig bekannt (vgl. Reisner 2016). Während sich die Forschung zu Geschlechtseffekten besonders auf die Jugendphase und das hohe Alter konzentriert, bleiben die anderen Lebensphasen eher unterrepräsentiert. Methodisch ist die Forschung in diesem Bereich relativ weit entwickelt und es gibt eine große Zahl an quantitativen Netzwerkstudien, darunter auch Längsschnittstudien mit SIENA-Modellen. Qualitative Netzwerkforschungen oder visuelle Netzwerkmethoden, die besonders für die Aufdeckung der Wirkweise von sozialen Netzwerken (siehe Kap.,Wirkmechanismen“) geeignet wären, findet man hingegen kaum. Eine Weiterentwicklung in diesem Bereich wäre lohnenswert.

Verglichen mit den Erwerbslosen und Alleinerziehenden sind Menschen mit Behinderung eine von der Netzwerkforschung weitgehend vernachlässigte Gruppe (siehe Kap. „Soziale Netzwerke und Behinderung“), obwohl sie mit 9,4\% einen verhältnismäßig großen Anteil der Gesamtbevölkerung stellen (Statistisches Bundesamt 2018). Diese Lücke ist umso gewichtiger, als dass Behinderungen erhebliche Konsequenzen für die Aufrechterhaltung und Bildung von Netzwerken und Beschäftigungsverhältnissen haben. Neben 
institutionellen Akteur*innen spielen viele andere Faktoren für die Gesunderhaltung dieser Gruppe eine Rolle, die selten mit Netzwerkparametern verknüpft werden (etwa regionale Zugänglichkeiten). Darüber hinaus verweisen auch Stefan Zapfel und Ko-Autor*innen auf die sich wandelnde Bedeutung verschiedener Institutionen.

Auch Migration ist, ebenso wie Erwerbslosigkeit, der Alleinerziehendenstatus oder Behinderung, keine „Krankheit“ (siehe Kap. „Migration als gesundheitliche Ungleichheitsdimension?"). Die in Zusammenhang mit Migration und natioethno-kultureller (Mehrfach-)Zugehörigkeit auftretenden Gesundheitsrisiken und assoziierte Netzwerkmechanismen - werden dabei über unterschiedliche Modelle, wie bspw. die „Healthy-migrant“-Hypothese, erklärt. Derartige Hypothesen werden forschungsmethodisch jedoch kaum verfolgt. Zudem werden andere Ungleichheitsdimensionen, wie ökonomisches oder kulturelles Kapital von Migrant*innen, kaum modelliert. Auch die Erfassung der Netzwerke von Migrant*innen weist, so die Autor*innen Annett Kupfer und Markus Gamper, noch erhebliche methodische Lücken auf. So fehlt es an Längsschnittstudien oder Studien mit Vergleichsgruppen. Negative Beziehungen (Kanten), die beispielsweise von Anfeindungen, Diskriminierungen oder Rassismus geprägt sein können, werden ebenso wenig wie sozialstaatliche oder institutionelle Knoten als Teil migrantischer Netzwerke erhoben. Schließlich liegen Studien, in denen die Faktoren Gesundheit, Netzwerke und Migration verbunden werden, kaum vor.

Die Autor*innen des dritten Teils des Bandes stellen übereinstimmend fest, dass die Mechanismen des Netzwerkeinflusses eher vorausgesetzt als untersucht werden. Dabei sind verschiedene Hypothesen denkbar, etwa der Verlust an Integration in die Kollegengruppe nach dem Übergang in die Erwerbslosigkeit oder der Zusammenhang von Unterstützung, negativen Beziehungen und Homogenität im Netzwerk bei Alleinerziehenden. Für alle Ungleichheiten gilt, dass in modernen Wohlfahrtsgesellschaften auch Institutionen maßgeblich am Gelingen der Netzwerkarbeit beteiligt sind, z. B. Jobcenter bei Erwerbslosigkeit, Familienhelfer*innen bei Alleinerziehenden, Integrationshelfer*innen bei Behinderten und Migrationsdienste bei Migrant*innen. Hier verschränken sich private und institutionelle Beziehungen, deren Zusammenwirken bisher jedoch kaum untersucht wurde (vgl. aber: Kupfer 2015). Auch für dieses Zusammenwirken lassen sich interessante Hypothesen bilden, deren Prüfung letztendlich auch die Funktionsmechanismen staatlicher Wohlfahrt untersucht. Beispielsweise könnten institutionelle und private Unterstützung sowohl in einem Verdrängungswettbewerb (ähnlich der These zu unterbrochenen Dyaden in der Entwicklung von Freundschaften nach Übergang zur Partnerschaft, siehe Kap. „Soziale Netzwerke und gesundheitliche Ungleichheiten im jungen und mittleren Erwachsenenalter") 
als auch in einer unterstützenden Beziehung zueinander stehen. Die unterschiedlichen Interaktionen ließen sich möglicherweise aus der Stärke der jeweiligen Beziehungen erklären. Bei der Verschränkung von staatlicher und privater Unterstützung bleibt fraglich, ob sich derartige Netzwerke tatsächlich verbinden oder ob definierte Grenzen innerhalb hybrider Netzwerkkonstruktionen bestehen bleiben. Derartige hybride Konstruktionen, in denen private und institutionelle Netzwerkteile verschränkt sind, würden sehr von der Einführung Kanten-spezifischer Mechanismen (beispielsweise Unterstützung in schwachen institutionellen Beziehungen, Ansteckung in starken proximalen Beziehungen) profitieren, ebenso wie von der Hinzunahme von negativen Beziehungen (etwa dem Misstrauen Alleinerziehender gegenüber dem Jugendamt bei gleichzeitiger Abhängigkeit von institutioneller Unterstützung). Wird der Gedanke der extensiven Netzwerkforschung und der Ausdehnung auf soziale Institutionen weiterverfolgt, so stellt sich die Frage nach der Darstellbarkeit von Netzwerkhierarchien und -strukturen, beispielsweise von „Netzwerken innerhalb von Netzwerken“. Ein Vorschlag für derartige Strukturen wurde von Reis (2017) vorgelegt, in dem er DDR-Familien als „Nischen“ innerhalb eines totalitären Staates beschrieb.

\section{$4 \quad$ Lebenslauf, Ungleichheit, Netzwerk, und Gesundheit - einige Hypothesen}

Zusammenfassend muss festgestellt werden, dass die Zusammenführung von Lebenslauf- und Ungleichheitsforschung für soziale Netzwerke und Gesundheit noch weitgehend aussteht. Lebensphasenübergreifende Netzwerkänderungen als Gesundheitsrisiken und -ressourcen zu begreifen, die entweder Folge (Mediation) von sozialen Ungleichheiten sind oder diese in ihrer Auswirkung beeinflussen (Moderation) - hier wartet ein neuer Forschungsbereich darauf, entwickelt zu werden. Mit der erweiterten Netzwerkperspektive, wie sie in der Einleitung dieses Bandes und am Anfang dieses Kapitels vorgestellt wurde, ließen sich viele bisher vermutete Zusammenhänge genauer prüfen, z. B.:

- Erfolg macht einsam und dann krank, aber nur wenn er aus einer sozial niederen Schicht entsteht und nach der Jugend beginnt.

- Gesunderhaltung von Jugendlichen ist zum großen Teil durch die Beziehungsarbeit ihrer Eltern bedingt, indem diese zum Beispiel negative Beziehungen abbauen (z. B. aus einer Risikonachbarschaft fortziehen), was jedoch nur denen gelingt, die über ausreichende Mittel verfügen. 
- Alter-Alter-Beziehungen beeinflussen Netzwerke in reicheren Schichten stärker als in ärmeren, womit Zugänge zum Gesundheitssystem erleichtert werden, wobei die Weichen hierfür schon während der Jugend gestellt werden.

- Mangelnde Pflegebereitschaft im hohen Alter ist oftmals das Resultat von negativen Beziehungen in früheren Zeiten, wobei „ärmere Alte“ schwerer betroffen sind als reichere.

- Gesundheitliches Risikoverhalten ist geeignet, die Position im Netzwerk zu verbessern (etwa die Zentralität), aber nur wenn das Gesamtnetz diese Möglichkeit erfolgreichen Vergleichens befördert - etwa, weil andere Netzwerkteilnehmer*innen besser mit Kapital ausgestattet sind. Derartige Netzwerkmechanismen bleiben auf die Jugend beschränkt, weil sich danach die Netzwerke sozial homogenisieren.

\section{$5 \quad$ Ausblick: Anforderungen an zukünftige Forschung}

Für die Formulierung von Desiderata für die zukünftige Forschung kommen wir noch einmal auf das anfangs vorgestellte Modell zurück (siehe Kap. „Soziale Netzwerke und gesundheitliche Ungleichheiten - eine neue Forschungsperspektive") und ordnen den dort beschriebenen Analyseebenen unsere Schlussfolgerungen $\mathrm{zu}$.

Für die oberste Modell-Ebene in Abb. 1 gilt nach wie vor die Forderung, dass soziale Ungleichheiten eine conditio sine qua non der Gesundheitsforschung sein sollten. Der zunehmende durchschnittliche Wohlstand der westlichen Industriestaaten ist aktuell mit wachsender sozialer Ungleichheit verbunden (Alvaredo et al. 2018), wobei sich beide Entwicklungen auf Gesundheit auswirken. Der soziale Wandel hin zur digitalen Wissensgesellschaft und -ökonomie (Reckwitz 2017) als Teil der „entfesselten Moderne“ (Giddens 2001) birgt erhebliche Risiken für Ungleichheit, Entbindung und Flexibilisierung mit sich, deren Umwandlung in Chancen hohe Aufwendungen, funktionale Netzwerke eingeschlossen, durch das Individuum verlangen. Kategorien von Ungleichheit, sowohl horizontale als auch vertikale, dynamisieren sich möglicherweise im Kontext digitaler Ökonomien. Mobilitäten, soziale wie räumliche, verlangen nach sozialem Kapital, deren Einschluss in Vorhersagemodelle für Gesundheit damit ein Standard werden sollte.

Die Objektivierung dieses sozialen Kapitals verlangt nach der möglichst genauen Messung von sozialen Netzwerken (Modell-Ebene 2 in Abb. 1). Die uneinheitliche und stellenweise nicht zielführende Messung von sozialen 


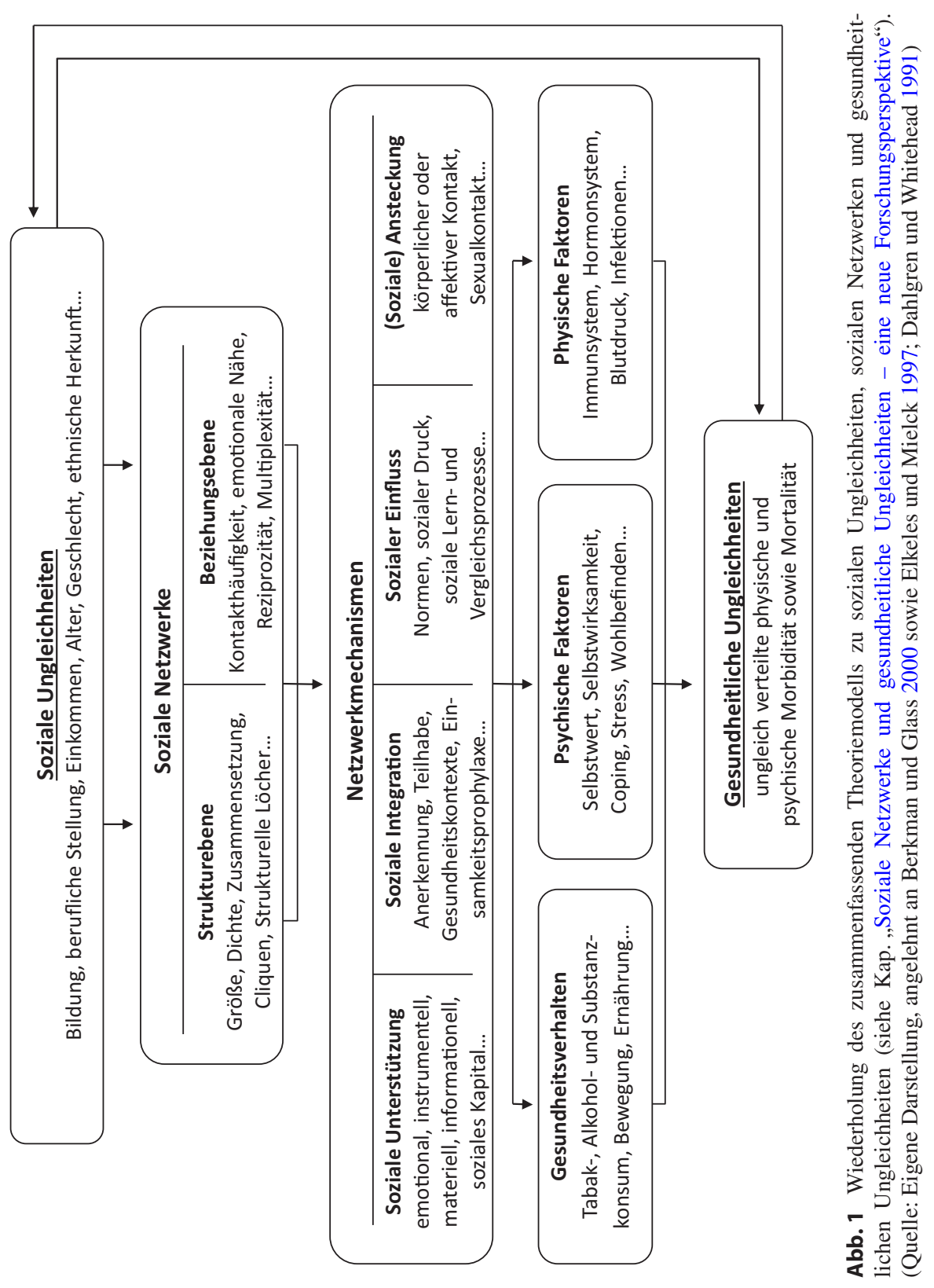


Netzwerken war ein Kritikpunkt, der in allen Beiträgen dieses Bandes geäußert wurde. Im Folgenden werden einige Forderungen an eine Netzwerkforschung aus den einzelnen Kapiteln zusammengetragen, die die angestrebte Mesofundierung von Gesundheit verbessern könnten:

- Das Netzwerk sollte über die Erfassung der Art und Anzahl von Kontakten hinausgehen und Aussagen über die Strukturebene ermöglichen. Hierzu gehören Parameter wie beispielsweise Dichte, Homogenität, Zentralität, Cliquen oder strukturelle Löcher.

- Das Netzwerk sollte weitergehende Informationen über die Alteri samt ihrer Eigenschaften beinhalten, sowohl als neu generierte Knoten (durch Akteursgeneratoren) als auch durch deren Beziehungen (Alter-Alter-Kanten). Hier liegen im deutschsprachigen Raum bereits erste ausgearbeitete Netzwerkmodule vor, die in großen Repräsentativerhebungen eingesetzt werden. Im ALLBUS etwa (siehe: www.gesis.de/allbus) werden zu den fünf wichtigsten Netzwerkpersonen die Alter-Alter-Relationen und zahlreiche Eigenschaften der Personen abgefragt. Das Soziooekonomische Panel (SOEP, siehe: www.diw.de/ soep) zielt eher auf die Netzwerkgröße von Individuen ab und nutzt dazu fünf Akteursgeneratoren mit je fünf Nennungsmöglichkeiten (d. h. einer Netzwerkgröße von maximal bis zu 20 Unterstützer*innen plus maximal bis zu fünf negative/belastende Beziehungen). Dazu kommen noch einige weitere Netzwerkfragen, die überwiegend auf die Größe und z. T. Qualität anderer Netzwerksektoren abzielen, ohne dass Alter-Alter-Kanten erfragt werden. Diese Module stellen jedoch wichtige und praktisch erprobte Ausgangspunkte für praktikable Netzwerkmodule zukünftiger Studien dar.

- Die Beziehungen selbst sollten grundsätzlich nicht nur gesundheitsförderliche (im Sinne von Unterstützung oder sozialem Kapital), sondern auch negative, gesundheitsbeeinträchtigende Aspekte beinhalten (wie es im SOEP bereits im Ansatz der Fall ist). Negative Beziehungen sind geeignet, gesundheitliches Vermögen zu reduzieren, und können sowohl im Nah- als auch im Fernraum des Individuums auftreten und direkt auf Ego wirken bzw. Alteri-Alteri-Beziehungen betreffen. Schließlich können Beziehungen prinzipiell unterstützend und belastend zugleich sein und sollten nicht auf eine (einzige) Funktion reduziert werden.

- Die Netzwerkerfassung sollte möglichst vergleichbar für verschiedene Kontexte sein, dies betrifft sowohl räumliche als auch zeitliche Dimensionen. Mit (sozial)räumlichen Dimensionen sind solche wie Stadt-Land, ArbeitFreizeit, offline-online oder Migrationsereignisse gemeint. Kulturell bedingte Frames - wie etwa unterschiedliche Definitionen von „Familie“, aber auch 
„Gesundheit“ - gehören ebenso hierher wie ereignisassoziierte Änderungen von Sozialräumen, etwa nach Arbeitslosigkeit oder im Leben mit einer Behinderung. Verschiedene Zeitkontexte meinen vor allem alters- und lebenslaufbedingte Netzwerkveränderungen, wobei in den Kapiteln des zweiten Teils dieses Bandes aufgezeigt wurde, dass unterschiedliche Lebensalter nicht nur mit unterschiedlichen Ereignisstrukturen assoziiert sind, sondern auch mit unterschiedlichen individuellen Voraussetzungen, Netzwerke zu gestalten. Grob gesagt, hat die individuelle Gestaltung des Netzwerkes über den Lebensverlauf die Form eines umgekehrten Us, womit sich sowohl Ausdehnung als auch Aktivitätsparameter im Lebensverlauf verändern. Räumliche und zeitliche Kontexte der Netzwerkerfassung stehen dabei in einem wechselseitigen Zusammenhang. Beispielsweise machen ,unzeitige“ Übergänge, d. h. nicht an die gesellschaftlichen Zeit-Normierungen angepasste Übergänge, - wie etwa eine verfrühte Elternschaft - das Betreten bestimmter Sozialräume - etwa der Universität - weniger wahrscheinlich. Ebenso sind die lebenslangen Risiken des Wechsels in die Erwerbslosigkeit unterschiedlich, je nachdem, wann und wie häufig dieser Übergang stattfindet, wobei hier der späte Übergang problematischer scheint.

- Mit der allgemein geltenden Reziprozitätsregel für soziale Beziehungen können dann aber auch zeitlich weit auseinanderliegende Netzwerkveränderungen aufeinander bezogen werden, wenn beispielsweise eine späte Arbeitslosigkeit der Eltern zur Beendigung des Arbeitslebens und gleichzeitig zu erhöhten Investitionen in die eigenen Kinder und Enkel führt - was möglicherweise gesundheitsförderliche Effekte des Familiennetzwerkes oder Unterstützung im Alter wahrscheinlicher macht.

- Gleichzeitig ist nicht davon auszugehen, dass Netzwerke im Sinne von Top-down-Prozessen nur auf passive Individuen einwirken, sondern gleichzeitig von diesen - bewusst, strategisch oder auch unbewusst - verändert werden, also Bottom-up-,,Kopplungen“ und Selektionseffekten unterliegen. Der aktive Beitrag des Einzelnen (etwa qua individueller Geselligkeit oder individuellem Netzwerken) ist eine wichtige zusätzliche Kontrollgröße.

- Eine ideale Netzwerkmessung sollte berücksichtigen, dass Netzwerke wahrscheinlich über unterschiedliche Mechanismen wirken (Modell-Ebene 3 in Abb. 1). Auch wenn sämtliche der von Klärner und von der Lippe (siehe Kap. „Wirkmechanismen in sozialen Netzwerken“) diskutierten Mechanismen in einer einzelnen Studie schwer abbildbar sein werden, sollte dennoch unterschieden werden, ob es sich um soziale Unterstützungs-, Einbindungs-, Einfluss- oder Ansteckungs-Effekte handelt. Hierfür bieten sich Kurzskalen an, wie sie beispielsweise mit der Oslo Social Support Scale (OSSS) (Dalgard 
et al. 2006) oder dem F-SozU K-14 (Fydrich et al. 2007) in validierter Form vorliegen. Während die OSSS soziale Unterstützung mit drei Items erfasst, steuern im F-SozU K-14 ebenfalls drei Items die Beschreibung der sozialen Integration einer Person bei.

- Hierbei sollten zukünftig die offline-Mechanismen in ihrer Verschränkung mit den online-Mechanismen untersucht werden, wobei sich von puren online-Makrostrategien - wie etwa dem berüchtigten Facebook-Experiment, in dem experimentell untersucht wurde, ob der emotionale Zustand der Nutzer*innen durch die Auswahl der im Newsfeed angezeigten Nachrichten manipuliert werden kann (Kramer et al. 2014; kritisch dazu: Kleinsman und Buckley 2015) - bis hin zu mikrosystemalen on/offline-Wechselverhalten etwa dem Übergang von (klassenübergreifenden) Multiplayer-Game-Netzwerken hin $\mathrm{zu}$ offline-Freundschaften alles denken lässt. Im mesosystemalen Bereich der sozialen Netzwerke sind inzwischen die größten Industrien der Neuzeit entstanden, deren Geschäftsmodelle eng an Mechanismen wie Einbindung, sozialen Einfluss oder Ansteckung gebunden sind. Hinter etlichen Knoten dieser Netzwerke stehen Maschinen oder Algorithmen, die sich nicht immer als solche zu erkennen geben. Damit dürften zukünftige Netzwerkstudien auch von den Forschungen zur Mensch-Mensch-Maschine-Interaktion (etwa bei partnerschaftlich geteilten Gesundheits-Apps) oder zur täuschenden Kommunikation profitieren.

Hier lassen sich diverse Hypothesen bilden, über welche Mechanismen soziale Netzwerke an proximale, d. h. mikrofundierte, Faktoren von Gesundheit ,koppeln“. Was die Messung auf dieser vierten Modellebene aus Abb. 1 betrifft, so findet auch hier augenblicklich eine Revolution statt, an der sich zukünftige Netzwerkforschung zu orientieren hat. Ohne diesen Gedanken weiter auszuführen, soll nur darauf verwiesen werden, dass sich beispielsweise Gesundheitsverhalten über sogenannte EMAs (ecological momentary assessment) von beispielsweise psychophysiologischen Parametern (Raugh et al. 2019) in hohem Maße objektivieren lässt. Psychische und physische Marker, vom Hautleitwiderstand, Blutdruck und Hirnaktivität bis hin zur aktuellen Genexpression für die Bildung des schlafsteuernden Hormons Melatonin, werden im Zuge von komplexer werdenden Analysen in den Gesundheitswissenschaften immer zugänglicher und können dynamisch (zeit- und ortsveränderlich) erfasst werden. Die Patient*innen der Zukunft müssen auf vielerlei Weise als verbunden (,,connected“) verstanden werden, womit offline- und online-Beziehungen zu den ,driving forces“ in der ,digitalen Psychiatrie“ und der „E-Health“ gehören (Bughra et al. 2017, S. 799). 
Auch die fünfte Ebene unseres vorgeschlagenen Theoriemodells, die Ungleichheit in psychischer und physischer Morbidität sowie Mortalität, unterliegt einem gewissen Wandel, zumindest was ihre Messung betrifft. So ändern sich Krankheitsdefinitionen beispielsweise mit der Einführung neuer Codiersysteme, wie der erwarteten Novellierung der ICD-10, womit $\mathrm{Zu}$ - oder Abnahmen in Krankheitsprävalenzen auch durch makrosystemale Änderungen in der Zuschreibung bedingt sein können. Der Meta-Text zu Krankheitskosten, wie sie beispielsweise durch das European Brain Council für Hirn-assoziierte Störungen im Sekundentakt bereitgestellt werden (https://www.braincouncil. $\mathrm{eu} /$ ), und ihrer Verteilung sollte in die Modellierung sozialer und gesundheitlicher Ungleichheiten einfließen. So bleibt es eine Forschungsfrage, ob und wie sich die Funktionen von Netzwerken in nach einem Versicherungsmodell finanzierten, in steuerfinanzierten, in rein privatwirtschaftlich finanzierten oder in mischfinanzierten Gesundheitssystemen unterscheiden.

\section{Netzwerk und gesundheitliche Ungleichheit: Hot topics}

Zum Ende sollen kaleidoskopartig einige Themen aufgelistet werden, die wir in diesem Band gern behandelt hätten, für die jedoch keine Zeit, kein Platz oder kein/e AutorIn zu gewinnen war. Wie Christakis und Fowler (2010) glauben auch wir, dass Netzwerke ein überall involviertes Agens menschlicher Entwicklung und damit der Gesundheit sind. Die folgende Aufzählung folgt daher keiner Systematik, sondern beschreibt nur Wünsche und Ideen der am Band beteiligten Forscher*innen.

\subsection{Pendeln, On- und Offline-Netzwerke, soziale Klasse}

Die erhebliche Flexibilisierung des Arbeitsmarktes in allen Sektoren, der zunehmende Teil von Leiharbeit und die Ausdehnung des Billiglohnsektors sind mit einer zunehmenden intra- und internationalen Arbeitsmigration verbunden, die die Beweglichkeit von Familien und convoys of life übersteigt (Rüger 2010). Die steigende Zahl von Arbeitspendler*innen verbringt zunehmend Zeit getrennt von offline-Netzen, wobei die Dauer der Arbeitsaufenthalte oft nicht hinreicht, um vor Ort offline-Netzwerke aufzubauen. Hier wäre z. B. zu fragen, inwieweit die mit der Migration verbundenen Gesundheitsrisiken durch online-Netzwerke moderiert werden, wie räumliche und soziale Mobilität verbunden sind, oder wie 
timing-Effekte, Netzwerk und sozialer Status zusammenhängen. Die Balance von online-offline-Kontakten kann in Abhängigkeit vom sozialen Status variieren, wenn z. B. manuellen Leiharbeitern (Beispiel: Erntehelfer*innen) weniger Ressourcen zur Verfügung stehen, ihre Familien regelmäßig zu sehen, als besser gestellten Leiharbeiter*innen aus der IT-Branche.

\subsection{Psychische Erkrankung, On- und Offline Netzwerke, soziale Klasse}

Grundsätzlich sind psychische Erkrankungen hoch mit dysfunktionalen sozialen Beziehungen vergesellschaftet, wobei diverse Wirkrichtungen sowohl in der Entstehung, Chronifizierung oder Therapie denkbar sind. Daneben waren die seit langem bekannten Zusammenhänge von sozialer Klasse und psychiatrischer Diagnose (Hollingshead und Redlich 1958) in den letzten Jahren selten Gegenstand wissenschaftlicher Forschung (Analyse Datenbank PsychInfo, März 2019) und verdienen es, im Rahmen der Netzwerkanalyse neu belebt zu werden. Ein Praxisbeispiel aus der Therapie soll diese Forderung illustrieren.

Die Behandlung psychischer Erkrankungen erfolgt in der Regel nur mit Einwilligung der Patient*innen. Die relative Isolation (von riskanten sozialen Kontexten) ist essenzieller Bestandteil vieler stationärer Therapien. Die allgemeine, elektronisch vermittelte Vernetzung über Raum- und Zeitgrenzen hinweg ist jedoch so normal geworden, dass viele Patient*innen zwar in eine Therapie, aber nicht in eine Netzabstinenz einwilligen. Viele Kliniken lassen daher zumindest zeitweilig die Kommunikation mit der „Außenwelt“ zu (Handy-Zeit), was ein Risiko für den Therapieerfolg darstellen kann. Beispielsweise wird die Therapie nicht-suizidalen selbstverletzenden Verhaltens mitunter konterkariert, wenn die Patient*innen über WhatsApp mit den Narrativen von Nicht-Patient*innen und Bildern zerschnittener Unterarme konfrontiert werden. $\mathrm{Zu}$ fragen wäre hier beispielsweise, wie Psychotherapie unter den Bedingungen ohne unterbrochene Dyaden gelingen kann, oder wie die Effekte von negativen Beziehungen minimiert werden können, oder wie eine Therapie unter Einbezug krankheitsrelevanter Plattformen verbessert werden kann. Analog zu den Ergebnissen für das jugendliche Rauchverhalten ließe sich beispielsweise vermuten, dass Jugendliche mit niedrigerem sozialen Status in ihren Netzwerken häufiger mit Risikoverhalten konfrontiert sind, da ihre Netzwerke weniger homophil sind. Beispielsweise sollte der Frage nachgegangen werden, ob sogenannte „Werther"- oder „Papageno“-Effekte (soziale Ansteckung suizidalen Verhaltens bzw. Ansteckung suizidpräventiven Verhaltens) unterschiedlich in verschiedenen 
sozialen Strata wirken. Es konnte beispielsweise gezeigt werden, dass mediale Vorbilder, über offline-Netzwerke vermittelt, auf suizidales Verhalten von Jugendlichen wirken (Abrutyn und Mueller 2014), ohne dass hier bisher gesundheitliche Ungleichheiten untersucht worden wären. Im Sinne der klassischen Studie von Hollingshead und Redlich (1958) bleibt auch die Frage bisher offen, ob die statusabhängigen Zugänge zum psychiatrischen Hilfesystem von Netzwerkfunktionen moderiert oder mediiert werden.

\subsection{Armut, Gesundheit und institutionelle Netzwerkbeziehungen}

Institutionen und professionelle Helfer*innen können, besonders für armutsgefährdete Personen, wichtige Akteure bzw. Knoten in einem Netzwerk sein (Klärner und Knabe 2016) und dort nicht nur einen Beitrag zum Wohlbefinden leisten, sondern - etwa durch Vermittlung präventiver oder kurativer Maßnahmen - auch stärker gesundheitsrelevante Wirkung entfalten. Die sozial ungleiche Verteilung und Barrieren des Zugangs in Abhängigkeit vom Wohnort und den Angeboten vor Ort können (gesundheitliche) Ungleichheiten verstärken (oder abmildern). Institutionelle Helfer*innen als Akteure einer sozialstaatlich bekämpften Armut (Paugam 2008) sind vorhanden und erreichbar oder eben nicht. $\mathrm{Zu}$ fragen wäre hier, in welcher Weise diese Akteure in einem sozialen Netzwerk wirken, ob sie Einfluss auf die Beziehungen zu anderen Akteuren haben, welche Netzwerkstrukturen das Wirken institutioneller Unterstützung förderlich werden lassen und welche, im Gegensatz dazu, eher Abhängigkeiten von diesen Akteuren hervorrufen und damit eher abträglich wirken und welche informellen Netzwerke tragen, wenn professionelle Hilfe nicht verfügbar ist.

\subsection{Die räumliche Dimension von sozialen Netzwerken: Gesundheit und soziale Netzwerke in ländlichen Räumen}

Soziale Netzwerke haben auch eine räumliche Dimension: Um direkte interpersonale Kontakte und Interaktionen mit Freund*innen, Bekannten, institutionellen Helfer*innen, Ärzt*innen etc. herzustellen oder bestimmte (Gesundheits-) Dienstleistungen in Kliniken, Apotheken oder durch Pflege- und Rettungsdienste 
in Anspruch zu nehmen, ist in der Regel eine räumliche Distanz zu überwinden. Gerade die Erreichbarkeit von Institutionen und Dienstleistern im Gesundheitsbereich ist räumlich und damit auch sozial ungleich verteilt (Neumeier 2017). $\mathrm{Zu}$ fragen wäre, welche Auswirkungen das Fehlen oder die schlechte Erreichbarkeit dieser institutionalisierten Knoten im Netzwerk hat, ob dies durch andere Bereiche des Netzwerkes abgefedert oder durch neue, digitalisierte Angebote ersetzt und ergänzt werden kann und welche Konsequenzen wiederum eine ungleich verteilte health literacy in diesem Zusammenhang hat. Erste Ansätze, netzwerkanalytische Methoden in diesem Zusammenhang fruchtbar zu machen und eine Form des räumlichen Kapitals (spatial capital) zu rekonstruieren, wurden auf einer von SoNegU organisierten Session bei der Sunbelt Social Network Conference vorgestellt (Galaskiewicz et al. 2016).

\section{Literatur}

Abrutyn, S., \& Mueller, A. S. (2014). Are suicidal behaviors contagious in adolescence? Using longitudinal data to examine suicide suggestion. American Sociological Review, 79, 211-227.

Alvaredo, F., Chancel, L., Piketty, T., Saez, E., \& Zucman, G. (Hrsg.). (2018). Die weltweite Ungleichheit. Der World Inequality Report 2018. München: Beck.

Berkman, L. F., \& Glass, T. (2000). Social integration, social networks, social support, and health. In L. F. Berkman \& I. Kawachi (Hrsg.), Social epidemiology (S. 137-173). New York: Oxford University Press.

Brand, T. (2018). Dimensionen gesundheitlicher Ungleichheit. In R. Haring (Hrsg.), Gesundheitswissenschaften (S. 1-13). Wiesbaden: Springer.

Bughra, D., Tasman, A., Pathare, S., Priebe, S., Smith, S., Torous, J., Arbuckle, M.R., ..., $\&$ Ventriglio, A. (2017). The WPA-Lancet psychiatry commission on the future of psychiatry. Lancet Psychiatry, 4(10), 775-818.

Christakis, N. A., \& Fowler, J. H. (2010). Connected! Die Macht sozialer Netzwerke und warum Glück ansteckend ist. Frankfurt a. M.: Fischer.

Dahlgren, G., \& Whitehead, M. (1991). Policies and strategies to promote social equity in health: Background document to WHO - Strategy paper for Europe. http://www. framtidsstudier.se/wp-content/uploads/2011/01/20080109110739filmZ8UVQv2wQFShMRF6cuT.pdf. Zugegriffen: 18. Apr. 2019.

Dalgard, O. S., Dowrick, C., Lehtinen, V., Vazquez-Barquero, J. L., Casey, P., Wilkinson, G., Ayuso-Mateos, J. L., Page, H., Dunn, G., \& The ODIN Group. (2006). Negative life events, social support and gender difference in depression: A multinational community survey with data from the ODIN study. Social Psychiatry and Psychiatric Epidemiology, 41, 444-451.

Elkeles, T., \& Mielck, A. (1997). Entwicklung eines Modells zur Erklärung gesundheitlicher Ungleichheit. Gesundheitswesen, 59, 137-143. 
Emirbayer, M. (1997). Manifesto for a relational sociology. American Journal of Sociology, 103(2), 281-317.

Fydrich, T., Sommer, G., \& Brähler, E. (2007). F-SozU: Fragebogen zur Sozialen Unterstützung. Manual. Göttingen: Hogrefe.

Galaskiewicz, J., Freeman Anderson, K., \& Thompson-Dyck, K. (2016). "SpatialCapital: Applications for the study of urban inequality.” Paper presented in the session on Social Networks and Health Inequalities. International Sunbelt Social Network Conference, April 5-10, Newport Beach, CA.

Giddens, A. (1984). Die Konstitution der Gesellschaft. Grundzüge einer Theorie der Strukturierung. Frankfurt a. M.: Campus.

Giddens, A. (2001). Entfesselte Welt. Wie die Globalisierung unser Leben verändert. Frankfurt a. M.: Suhrkamp.

Hollingshead, A. B., \& Redlich, F. C. (1958). Social class \& mental illness. New York: Wiley.

Klärner A., \& Keim, S. (2019). Emirbayer (1997). Manifesto for a Relational Sociology. In B. Holzer \& C. Stegbauer (Hrsg.), Schlüsselwerke der Netzwerkforschung (S. 177-180). Wiesbaden: Springer VS.

Klärner, A., \& Knabe, A. (2016). Soziale Netzwerke als Ressource für den Umgang mit den Folgen von Langzeitarbeitslosigkeit. WSI-Mitteilungen, 69(5), 353-364.

Kleinsman, J., \& Buckley, S. (2015). Facebook study: A little bit unethical but worth it? Bioethical Inquiry, 12(2), 179-182.

Kramer, A. D. I., Guillory, J. E., \& Hancock, J. T. (2014). Experimental evidence of massive-scale emotional contagion through social networks. Proceedings of the National Academy of Sciences, 111(24), 8788-8790.

Kupfer, A. (2015). Wer hilft helfen? Einflüsse sozialer Netzwerke auf Beratung. Tübingen: dgvt.

Lüscher, K., \& Pillemer, K. (1998). Intergenerational ambivalence: A new approach to the study of parent-child relations in later life. Journal of Marriage and the Family, 60, $413-425$.

Neumeier, S. (2017). Regionale Erreichbarkeit von ausgewählten Fachärzten, Apotheken, ambulanten Pflegediensten und weiteren ausgewählten Medizindienstleistungen in Deutschland - Abschätzung auf Basis des Thünen-Erreichbarkeitsmodells (Thünen Working Paper 77). Braunschweig: Johann Heinrich von Thünen Institut.

Paugam, S. (2008). Die elementaren Formen der Armut. Hamburg: Hamburger Edition.

Raugh, I. M., Chapman, H. C., Bartolomeo, L. A., Gonzalez, C., \& Strauss, G. P. (2019). A comprehensive review of psychophysiological applications for ecological momentary assessment in psychiatric populations. Psychological Assessment, 31(3), 304-317.

Reckwitz, A. (2017). Die Gesellschaft der Singularitäten. Zum Strukturwandel der Moderne. Berlin: Suhrkamp.

Reis, O. (2017). Nischen im Wandel. Zur Transformation von Familien und Generationenbeziehungen in Ostdeutschland. Gießen: Psychosozial.

Reisner, S. L., Poteat, T., Keatley, J., Cabral, M., Mothopeng, T., Dunham, E., Holland, C. E., Max, R., \& Baral, S. D. (2016). Global health burden and needs of transgender populations: A review. The Lancet, 388(10042), 412-436. 
Rüger, H. (2010). Berufsbedingte räumliche Mobilität in Deutschland und die Folgen für Familie und Gesundheit. Bevölkerungsforschung Aktuell, 31(2), 8-12.

Statistisches Bundesamt. (2018). Statistik der schwerbehinderten Menschen 2017. Kurzbericht. https://www.destatis.de/DE/Themen/Gesellschaft-Umwelt/Gesundheit/BehinderteMenschen/Publikationen/Downloads-Behinderte-Menschen/sozial-schwerbehinderte-kb-5227101179004.pdf. Zugegriffen: 10. Mai 2019.

Open Access Dieses Kapitel wird unter der Creative Commons Namensnennung 4.0 International Lizenz (http://creativecommons.org/licenses/by/4.0/deed.de) veröffentlicht, welche die Nutzung, Vervielfältigung, Bearbeitung, Verbreitung und Wiedergabe in jeglichem Medium und Format erlaubt, sofern Sie den/die ursprünglichen Autor(en) und die Quelle ordnungsgemäß nennen, einen Link zur Creative Commons Lizenz beifügen und angeben, ob Änderungen vorgenommen wurden.

Die in diesem Kapitel enthaltenen Bilder und sonstiges Drittmaterial unterliegen ebenfalls der genannten Creative Commons Lizenz, sofern sich aus der Abbildungslegende nichts anderes ergibt. Sofern das betreffende Material nicht unter der genannten Creative Commons Lizenz steht und die betreffende Handlung nicht nach gesetzlichen Vorschriften erlaubt ist, ist für die oben aufgeführten Weiterverwendungen des Materials die Einwilligung des jeweiligen Rechteinhabers einzuholen.

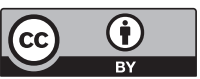

